\title{
Article \\ Symmetries of Thirring Models on 3D Lattices
}

\author{
Andreas W. Wipf *(1) and Julian J. Lenz (1) \\ Theoretisch-Physikalisches-Institute, Friedrich-Schiller-Universität, Max Wien Platz 1, 07743 Jena, Germany; \\ julian.johannes.lenz@uni-jena.de \\ * Correspondence: wipf@tpi.uni-jena.de
}

\section{check for}

updates

Citation: Wipf, A.W.; Lenz, J.J.

Symmetries of Thirring Models on

3D Lattices. Symmetry 2022, 14, 333.

https://doi.org/10.3390/sym14020333

Academic Editors: Simon Hands and

Simon Catterall

Received: 1 January 2022

Accepted: 1 February 2022

Published: 6 February 2022

Publisher's Note: MDPI stays neutral with regard to jurisdictional claims in published maps and institutional affiliations.

Copyright: () 2022 by the authors Licensee MDPI, Basel, Switzerland. This article is an open access article distributed under the terms and conditions of the Creative Commons Attribution (CC BY) license (https:// creativecommons.org/licenses/by/ $4.0 /)$.

\begin{abstract}
We review some recent developments about strongly interacting relativistic Fermi theories in three spacetime dimensions. These models realize the asymptotic safety scenario and are used to describe the low-energy properties of Dirac materials in condensed matter physics. We begin with a general discussion of the symmetries of multi-flavor Fermi systems in arbitrary dimensions. Then we review known results about the critical flavor number $N_{\text {crit }}$ of Thirring models in three dimensions. Only models with a flavor number below $N_{\text {crit }}$ show a phase transition from a symmetry-broken strong-coupling phase to a symmetric weak-coupling phase. Recent simulations with chiral fermions show that $N_{\text {crit }}$ is smaller than previously extracted with various non-perturbative methods. Our simulations with chiral SLAC fermions reveal that for four-component flavors $N_{\text {crit }}=0.80(4)$. This means that all reducible Thirring models with $N_{\mathrm{r}}=1,2,3, \ldots$ show no phase transition with order parameter. Instead, we discover footprints of phase transitions without order parameter. These new transitions are probably smooth and could be used to relate the lattice Thirring models to Thirring models in the continuum. For a single irreducible flavor, we provide previously unpublished values for the critical couplings and critical exponents.
\end{abstract}

Keywords: model field theory; chiral symmetry breaking; parity breaking; dynamical fermions; four-Fermi theories; Thirring model

\section{Introduction}

The Thirring model is a relativistic field theory for interacting fermions $\bar{\psi}, \psi$. Its vector-vector interaction,

$$
\mathcal{L}_{\text {int }}=\frac{g^{2}}{2}\left(\bar{\psi} \gamma^{\mu} \psi\right)^{2}=\frac{g^{2}}{2} J^{\mu} J_{\mu}
$$

establishes (after bosonization) a close relation to QED but it is also studied in various other contexts, for example, as a test bed for non-perturbative methods, as an example of asymptotic safety or as a toy model for chirally symmetric fermions. The coupling constant $g^{2}$ has length-dimension $(d-2)$ and is dimensionless in 2 dimensions.

The 2-dimensional massless model was introduced and investigated by Walter Thirring in 1958 [1] and represents an exactly solvable conformal field theory with analytically known $n$-point correlation functions [2,3]. The massive model can be solved with the Bethe ansatz, which yields the mass spectrum and scattering matrix elements. Above 2 dimensions, the model is not soluble and not renormalizable in perturbation theory. However, it is renormalizable beyond perturbation theory-above 2 and below 4 dimensions, it is the prototype of an asymptotically safe theory [4].

We begin with discussing the symmetries of Thirring models with Lagrangian

$$
\mathcal{L}=\mathcal{L}_{m}+\frac{g^{2}}{2 N_{\mathrm{f}}} J^{\mu} J_{\mu}, \quad \mathcal{L}_{m}=\sum_{a} \bar{\psi}_{a}(\not \partial+m) \psi_{a},
$$

for $N_{\mathrm{f}}$ flavors of fermions $\psi_{1}, \ldots, \psi_{N_{\mathrm{f}}}$ in arbitrary dimensions. Then we shall discretize the models on a (euclidean) spacetime lattice with chiral fermions keeping all continuum symmetries besides Poincare invariance. We shall focus on the lattice models in 3 dimensions 
and discuss the possible breaking of symmetries, depending on the number of flavors and the interaction strength. Finally, we summarize the present status concerning the critical flavor number $N_{\mathrm{f}}^{\text {crit }}$ which separates the systems with spontaneous parity breaking from those without symmetry breaking.

An irreducible spinor in $d$-dimensions has $d_{s}=2^{\lfloor d / 2\rfloor}$ components, where $\lfloor a\rfloor$ is the largest integer less than or equal to $a$. In euclidean space we can and will always choose hermitian $\gamma^{\mu}$ matrices. In even dimensions there exists one irreducible representation of the Clifford algebra, whereas in odd dimensions there are two. The hermitean matrix,

$$
\gamma_{*}=-\mathrm{i}^{\lfloor d / 2\rfloor} \gamma^{0} \cdots \gamma^{d-1}, \quad \gamma_{*}^{2}=\mathbb{1}
$$

generalizes $\gamma_{5}$ to arbitrary dimensions. In even dimensions it anticommutes with the $\gamma^{\mu}$ and in odd dimensions it commutes with the $\gamma^{\mu}$ and is $\mathbb{1}$ in one irreducible representation and $-\mathbb{1}$ in the other irreducible representation. The bilinear fields,

$$
S=\sum_{a=1}^{N_{\mathrm{f}}} \bar{\psi}_{a} \psi_{a}, \quad P=\mathrm{i} \sum_{a=1}^{N_{\mathrm{f}}} \bar{\psi}_{a} \gamma_{*} \psi_{a}, \quad J^{\mu}=\sum_{a=1}^{N_{\mathrm{f}}} \bar{\psi}_{a} \gamma^{\mu} \psi_{a},
$$

are of particular interest here: the current density $J^{\mu}$ enters the Lagrangian of the Thirring models and $S, P$ may or may not condense in an equilibrium state. One should note that $S$ and $P$ are not independent in odd dimensions because $\gamma_{*} \propto \mathbb{1}$ is trivial.

\section{Symmetries of Fermi Systems}

The symmetries of Thirring models with Lagrangians (2) are the usual (Euclidean) spacetime symmetries (including parity), chiral rotations and charge conjugation.

Charge conjugation:

The transformation of spinor fields and $\gamma^{\mu}$-matrices under charge conjugation,

$$
\psi_{c}=\mathcal{C} \psi^{*}, \quad \gamma_{\mu}^{T}=\eta_{\mathcal{C}} \mathcal{C}^{-1} \gamma_{\mu} \mathcal{C}, \quad \eta_{c} \in\{-1,1\}
$$

are used to investigate the sign problem in four-Fermi theories. Actually there are two matrices $\mathcal{C}$ with $\eta_{c}= \pm 1$ in even dimensions, one $\mathcal{C}$ with $\eta_{c}=-1$ in $3+4 n$ dimensions and one $\mathcal{C}$ with $\eta_{\mathcal{c}}=1$ in $1+4 n$ dimensions for $n \in \mathbb{N}_{0}$ [5].

Parity:

A parity transformation flips the sign of a spatial coordinate, for example,

$$
x \mapsto x^{\prime}=P x, \quad P=\left(P_{v}^{\mu}\right)=\operatorname{diag}(1,1, \ldots, 1,-1),
$$

Scalar and pseudo-scalar fields have even and odd parity, respectively. A spinor and its conjugate transform according to:

$$
\psi(x) \mapsto \psi_{P}\left(x^{\prime}\right)=\mathcal{P} \psi(x), \quad \bar{\psi}(x) \mapsto \bar{\psi}_{P}\left(x^{\prime}\right)=\alpha \bar{\psi}(x) \mathcal{P}^{-1}
$$

where the parity matrix $\mathcal{P}$ satisfies:

$$
\mathcal{P}^{-1} \gamma^{\mu} \mathcal{P}=\alpha P^{\mu}{ }_{\nu} \gamma_{v} \Longrightarrow \mathcal{P}^{-1} \gamma_{*} \mathcal{P}=-\alpha^{d} \gamma_{*}, \quad \alpha^{2}=1
$$

The density $\mathcal{L}_{m=0}$ is parity invariant, $J^{\mu}$ is a vector and $P$ is a pseudoscalar,

$$
P_{P}\left(x^{\prime}\right)=-P(x) \text {. }
$$

The transformation properties of $S$ depend on the dimension, that is,

$$
S_{P}\left(x^{\prime}\right)=\alpha S(x),
$$


such that $S$ is a scalar in even dimensions while $S \propto P$ is pseudoscalar in odd dimensions as detailed below.

\subsection{Even Dimension}

In even dimensions, there is only one irreducible representation of the Clifford algebra and there exists a canonical choice for the free Lagrangian $\mathcal{L}_{m}$ with the same $\not \partial$ acting on all flavors. For a vanishing mass the Dirac operator $\not \partial$ anti-commutes with $\gamma_{*}$ and we may rotate the chiral (left- and right-handed) fermions,

$$
\psi_{ \pm}=P_{ \pm} \psi=\frac{1}{2}\left(\mathbb{1} \pm \gamma_{*}\right) \psi,
$$

independently among each other,

$$
\psi_{+} \mapsto U_{+} \psi_{+}, \quad \psi_{-} \mapsto U_{-} \psi_{-} \quad U_{+}, U_{-} \in \mathrm{U}\left(N_{\mathrm{f}}\right) .
$$

These chiral rotations leave the Lagrangian of massless fermions $\mathcal{L}_{m=0}$ invariant. The bilinear $\propto \bar{\psi} \psi$ is only invariant under the diagonal subgroup with $U_{+}=U_{-}$. Thus, a mass term or a condensate $\langle\bar{\psi} \psi\rangle$ break the chiral symmetry explicitly, respectively and spontaneously to the vector flavor symmetry $\mathrm{U}_{V}\left(N_{\mathrm{f}}\right)$.

In even dimensions, there is a parity matrix $\mathcal{P}$ with $\alpha=1$ such that the bilinear $S$ is a scalar and $P$ a pseudoscalar, see (9). In addition, there exist two matrices $\mathcal{C}$, which obey (5), one for each sign of $\eta_{c}$.

\subsection{Odd Dimensions}

In odd dimensions, there is no notion of chirality in an irreducible representation and there are only the vector flavor rotations,

$$
\psi \mapsto U \psi, \quad \bar{\psi} \rightarrow \bar{\psi} U^{\dagger}, \quad U \in \mathrm{U}\left(N_{\mathrm{f}}\right),
$$

which leave $\mathcal{L}_{m}$ in (2) invariant. The bilinears (4) are singlets under these rotations. In odd dimensions there exists a parity matrix $\mathcal{P}$ which fulfills (8) with $\alpha=-1$ such that the bilinear $S$ is parity-odd [5],

$$
S_{P}\left(x^{\prime}\right)=-S(x) .
$$

Actually, the bilinears $S$ and $P$ are not independent, $S= \pm \mathrm{i} P$, since $\gamma_{*}= \pm \mathbb{1}$ in the two irreducible representations of the Clifford algebra. We see that a mass term or bilinear condensate break the $\mathbb{Z}_{2}$-parity symmetry explicitly or spontaneously.

The last statement applies to systems with odd $N_{\mathrm{f}}$ only, as for even $N_{\mathrm{f}}$ we can build a parity invariant massive Lagrangian. For example, for $N_{\mathrm{f}}=2$ one combines the two irreducible flavors to one reducible flavor and acts with the inequivalent irreducible representations $\gamma^{\mu}$ and $-\gamma^{\mu}$ on the upper and lower components,

$$
\mathcal{L}_{m}=\bar{\Psi}\left(\Gamma^{\mu} \partial_{\mu}+m\right) \Psi, \quad \Psi=\left(\begin{array}{l}
\psi_{1} \\
\psi_{2}
\end{array}\right), \quad \Gamma^{\mu}=\sigma_{3} \otimes \gamma^{\mu}
$$

For the reducible system $\tilde{\mathcal{P}}=\mathrm{i} \sigma_{2} \otimes \mathcal{P}$ is a parity matrix satisfying the defining relation (8) with $\Gamma^{\mu}$ and $\alpha=1$ such that $\bar{\Psi} \Psi$ and hence $\mathcal{L}_{m}$ in (15) are parity even. This construction straightforwardly generalizes to an even flavor number $N_{\mathrm{f}}=2 N_{\mathrm{r}}$. One just groups the $2 N_{\mathrm{r}}$ irreducible flavors into $N_{\mathrm{r}}$ reducible flavors $\Psi_{1}, \ldots, \Psi_{N_{\mathrm{r}}}$. The Lagrangian for the latter reads. (we also rescale the coupling such that $g^{2} / 2 N_{\mathrm{f}} \rightarrow g^{2} / 2 N_{\mathrm{r}}$ )

$$
\mathcal{L}_{m}=\sum_{a=1}^{N_{\mathrm{r}}} \bar{\Psi}_{a}\left(\Gamma^{\mu} \partial_{\mu}+m\right) \Psi_{a}+\frac{g^{2}}{2 N_{\mathrm{r}}} J^{\mu} J_{\mu}, \quad J^{\mu}=\sum_{a} \bar{\Psi}_{a} \Gamma^{\mu} \Psi_{a} .
$$

By construction, this parity invariant reducible model is invariant under $\mathrm{U}\left(N_{\mathrm{f}}\right)=\mathrm{U}\left(2 N_{\mathrm{r}}\right)$ rotations. $\mathcal{L}_{m}$ can be obtained by a dimensional reduction of the Thirring model in one dimension higher. The various symmetries of reducible four-Fermi systems are well explained in $[4,6]$. 


\subsection{Fierz-Identities}

It may happen that two seemingly different looking four-Fermi theories are equivalent on account of Fierz-identities. In 2 and 3 dimensions irreducible spinors have 2 components only and there is a direct way to relate different one-flavor models. Indeed, for a 2-component anti-commuting $\psi$ an arbitrary four-Fermi term $(\bar{\psi} A \psi)^{2}$ at a fixed spacetime point must be proportional to $\bar{\psi}_{1} \psi_{1} \bar{\psi}_{2} \psi_{2}$ and

$$
(\bar{\psi} A \psi)^{2}=\operatorname{det} A(\bar{\psi} \psi)^{2}, \quad N_{\mathrm{f}}=1, \quad d=2,3 .
$$

1. One-flavor models in two and three dimensions: The hermitian $2 \times 2$ matrices $\gamma^{\mu}$ have determinant -1 and we conclude

$$
\frac{g^{2}}{2}\left(\bar{\psi} \gamma^{\mu} \psi\right)^{2}=-\frac{d g^{2}}{2}(\bar{\psi} \psi)^{2}, \quad N_{\mathrm{f}}=1, \quad d=2,3 .
$$

It follows that the one-flavor Thirring model is equivalent to the one-flavor GrossNeveu (GN) model. Thus the latter is not only invariant under $\mathrm{U}(1) \times \mathbb{Z}_{2}$ but also under the larger symmetry group $\mathrm{U}_{V}(1) \times \mathrm{U}_{A}(1)$.

2. A comparable simple relation does not exist for $N_{\mathrm{f}}>1$ or $d>3$. For example, the general Fierz identity in 3 dimensions implies that, for $N_{\mathrm{f}}$ irreducible flavors,

$$
\frac{g^{2}}{2}\left(\bar{\psi} \gamma^{\mu} \psi\right)^{2}=-\frac{g^{2}}{2 N_{\mathrm{f}}}(\bar{\psi} \psi)^{2}-\frac{g^{2}}{N_{\mathrm{f}}} \sum_{a, b}\left(\bar{\psi}^{a} \psi^{b}\right)\left(\bar{\psi}^{b} \psi^{a}\right), \quad d=3 .
$$

This means that the Thirring interaction is converted into a GN interaction plus a tensor-tensor coupling.

\subsection{Hubbard-Stratonovich Transformation}

It is possible to eliminate the four-Fermi terms in the Lagrangian by a HubbardStratonovich transformation with the help of an auxiliary vector field $v_{\mu}$,

$$
\mathcal{L}=\bar{\psi} \mathcal{D}_{m} \psi+\frac{N_{\mathrm{f}}}{2 g^{2}} v_{\mu} v^{\mu} .
$$

The Dirac operator contains the auxiliary field,

$$
\mathcal{D}_{m}=\gamma^{\mu}\left(\partial_{\mu}+\mathrm{i} v_{\mu}\right)+m .
$$

The classical systems with Lagrangians (20) and (2) are equivalent as follows from the field equation for the auxiliary vector field. The equivalence also holds for the quantized system, since $v_{\mu}$ is non-dynamical, enters the Lagrangian at most quadratically and thus can be integrated over explicitly in the path integral.

In passing we note that the equivalent one-flavor GN-model with interaction term (18) in $d=2,3$ can be bosonized with a scalar field $\sigma$ as

$$
\mathcal{L}=\bar{\psi}(\not \partial+m+\sigma) \psi+\frac{1}{2 d g^{2}} \sigma^{2} .
$$

Interestingly, the fermion determinant of $\not \partial+\sigma$ is (generically) complex, whereas that of $\mathcal{D}_{0}$ is real. This means that by a Fierz-reshuffling from the scalar into the vector channel one can soften or even solve the ubiquitous sign problem.

\section{Critical Flavor Number of 3D Thirring Models}

In the following we focus on the 3-dimensional multi-flavor Thirring model. The irreducible systems have Lagrangian (2) for a multiplet $\psi$ of $N_{\mathrm{f}}$ two-component spinor fields and the reducible ones have Lagrangian (16) for a multiplet of $N_{\mathrm{r}}$ four-component reducible fields. There are several reasons for considering the subclass of reducible Thirring models. 
First and most important, most applications in condensed matter physics deal with Diractype materials where naturally one is lead to the parity-invariant reducible models. The most prominent example is of course graphene, where low-energy electronic excitations exhibit a linear dispersion around two Dirac points in the first Brillouin zone. Second, most results in the literature are obtained for the reducible systems, and for an ease of comparison we consider such systems as well. The reducible systems are obtained by a torus-reduction of four-dimensional interacting Fermi systems.

Monte Carlo simulations may suffer from a "negative sign problem" when applied to fermions. This could happen when the fermion determinant changes sign or becomes complex causing an exponential increase of the computing time with the number of particles $[7,8]$. Fortunately, the reducible Thirring models, in contrast to the irreducible ones with odd $N_{\mathrm{f}}$, have no such sign problem. For example, for one reducible flavor $\Psi$, the fermion determinant of $\mathcal{D}_{m}^{\text {red }}$ in

$$
\mathcal{L}_{\text {red }}=\bar{\Psi} \mathcal{D}_{m}^{\text {red }} \Psi+\frac{1}{2 g^{2}} v_{\mu} v^{\mu}, \quad \mathcal{D}_{m}^{\text {red }}=\left(\begin{array}{cc}
\mathcal{D}_{m} & 0 \\
0 & -\mathcal{D}_{-m}
\end{array}\right)
$$

is $\operatorname{det}\left(m^{2}-\mathcal{D}_{0}^{2}\right)>0$, since $\mathcal{D}_{0}=\mathcal{D}_{m=0}$ defined in (21) is anti-hermitean. Note that the massive reducible model with $N_{\mathrm{r}}$ flavors is not equivalent to the irreducible model with $2 N_{\mathrm{r}}$ flavors. The two irreducible flavors have opposite mass and this explains why there is no breaking of parity in the reducible model. Only in the limit $m \rightarrow 0$ are the two models equivalent. The passage from one to the other involves a relative rotations of the two irreducible flavors, which combine to a reducible flavor such that:

$$
\mathcal{D}_{0}^{\text {red }} \mapsto\left(\begin{array}{cc}
\mathcal{D}_{0} & 0 \\
0 & \mathcal{D}_{0}
\end{array}\right)
$$

At the same time, the chiral condensate of one formulation transforms into the staggered condensate in the other:

$$
\langle\bar{\Psi} \Psi\rangle=\left\langle\bar{\psi}_{1} \psi_{1}\right\rangle-\left\langle\bar{\psi}_{2} \psi_{2}\right\rangle,
$$

and vice versa.

Small and Large- $N_{\mathrm{f}}$ Limit

Integrating over the fermion fields in the (euclidean) functional integral with Lagrangian (20) yields

$$
Z=\int \mathcal{D} v_{\mu} e^{-N_{\mathrm{f}} S_{\text {eff }}\left[v_{\mu}\right]}, \quad S_{\text {eff }}=\frac{1}{2 g^{2}} \int \mathrm{d}^{3} x v_{\mu}^{2}-\frac{1}{2} \log \operatorname{det}\left(-\mathcal{D}_{m}^{2}\right) .
$$

In the large- $N_{\mathrm{f}}$ limit the absolute minimum of $S_{\text {eff }}$ dominates the path integral such that the free energy per flavor simplifies considerably,

$$
F=-\frac{1}{\beta N_{\mathrm{f}}} \log Z \stackrel{N_{\mathrm{f}} \rightarrow \infty}{\longrightarrow} \frac{1}{\beta} \min _{v_{\mu}} S_{\mathrm{eff}}\left[v_{\mu}\right] .
$$

The Euler-Lagrange equation for $S_{\text {eff }}$ is just the gap equation which determines the minimizing field $v_{\mu}$. For a translation-invariant equilibrium state the minimizing field is homogeneous and for a constant $v^{\mu}$ the eigenvalues of $\mathcal{D}_{0}$ come in pairs $\pm \lambda$ which implies

$$
\operatorname{det}\left(-\mathcal{D}_{m}^{2}\right)=\operatorname{det}\left(\mathcal{D}_{m}^{\text {red }}\right) .
$$


This means that in the large- $N_{\mathrm{f}}$ limit the irreducible and the parity invariant reducible systems are identical, or that the irreducible models do not break parity. This observation is supported by an explicit calculation of the free energy density (effective potential):

$$
U_{\mathrm{eff}}=\frac{F}{V}=\frac{1}{2 g_{\text {ren }}^{2}} v_{\mu} v^{\mu}+U_{\text {free }}\left(T, m^{2}\right), \quad g^{2}=\frac{4 \pi g_{\text {ren }}^{2}}{4 \pi+\Lambda g_{\text {ren }}^{2}},
$$

where $\Lambda$ is the momentum cutoff. Besides the free energy density of the free Fermi gas one only gets a renormalization of the Thirring coupling. The parity condensate is obtained by differentiating $U_{\text {eff }}$ with respect to the trigger mass $m$. Since the derivative does not depend on the auxiliary field we conclude

$$
\langle\bar{\psi} \psi\rangle=\frac{\partial}{\partial m} U_{\text {free }} \stackrel{m \rightarrow 0}{\longrightarrow} 0, \quad N_{\mathrm{f}} \rightarrow \infty
$$

Thus, there is no parity condensate for a large number of flavors.

In the other limit $N_{\mathrm{f}}=1$ the Thirring model is equivalent to the 3-dimensional GrossNeveu model, see Section 2.4, and the latter shows spontaneous breaking of parity [9]. Thus we have:

$$
\langle\bar{\psi} \psi\rangle \neq 0, \quad N_{\mathrm{f}}=1 .
$$

Since parity is broken for $N_{\mathrm{f}}=1$ and unbroken for $N_{\mathrm{f}} \rightarrow \infty$ we must conclude that there exists a critical flavor number $N_{\mathrm{f}}^{\text {crit }}$ separating the systems with symmetry breaking from those without symmetry breaking.

For reasons explained above, the parity invariant reducible models are of particular interest. In the limit $N_{\mathrm{f}} \rightarrow \infty$ they are identical to the irreducible Thirring models. Since the parity breaking model with one irreducible flavor is not in the class of reducible modelsformally it has 0.5 reducible flavors - there is no compelling argument that there must exist a critical flavor number $N_{r}^{\text {crit }}$ within the class of reducible models.

First investigations of Thirring models with Schwinger-Dyson equations, partly in combination with a large- $N_{\mathrm{f}}$ expansion, date back to the nineties of the last century. In Table 1 we collected values for the critical flavor numbers $N_{\mathrm{f}}^{\text {crit }}$ and $N_{\mathrm{r}}^{\text {crit }}$ obtained with Schwinger-Dyson (SD) equations or expansions in $1 / N_{\mathrm{f}}$, a Gaussian ansatz for the state of interest in the Schrödinger picture, the functional renormalization group (FRG) and dedicated lattice simulations.

Table 1. Critical flavor numbers $N_{\mathrm{f}}^{\text {crit }}$ and $N_{\mathrm{r}}^{\text {crit }}$. SD means Schwinger-Dyson and FRG functional renormalization group. For example, SD-equations predicted that for $N_{\mathrm{f}}=1,2,3$ there is a parity condensate $\langle\bar{\psi} \psi\rangle$ and simulations with staggered fermions that for $N_{\mathrm{r}}=1,2, \ldots, 6$ there is a condensate $\langle\bar{\Psi} \Psi\rangle$.

\begin{tabular}{lccrr}
\hline Method & $N_{\mathbf{f}}^{\text {crit }}$ & $N_{\mathbf{r}}^{\text {crit }}$ & References & Years \\
\hline SD equations & 6.48 & 3.24 & {$[10]$} & 1991 \\
& $\infty$ & & {$[11]$} & 1994 \\
& & 4.32 & {$[12,13]$} & 1995,1997 \\
\hline $1 / N_{\mathrm{f}}$-expansion & & 2.00 & {$[14]$} & 1995 \\
& $<3$ & & {$[15]$} & 1998 \\
\hline Gaussian approximation & $\infty$ & $\infty$ & {$[16]$} & 1994 \\
\hline FRG & & 5.1 & {$[17]$} & 2012 \\
& & $\lesssim 2$ & {$[18]$} & 2019 \\
\hline lattice (staggered) & & $(4 \ldots 6)$ & {$[19,20]$} & 1997,1999 \\
& $\lesssim 9$ odd & 0.61 & {$[21,22]$} & 1999,2007 \\
\hline lattice (slac) & & $(1 \ldots 2)$ & {$[23,24]$} & 2017,2019 \\
\hline lattice (domain wall) & & & {$[25,26]$} & 2018,2020 \\
\hline
\end{tabular}


Early lattice studies were performed with light staggered fermions to recover the chiral symmetry in the continuum limit. With the help of an HMC algorithm, simulations with an even $N_{\mathrm{r}}$ and subsequently with non-integer $N_{\mathrm{r}}$ have been presented in [20,21]. In a subsequent lattice study [22] with a similar setup, the authors concluded that the critical flavor number is $N_{\mathrm{r}}^{\text {crit }}=6.6(1)$.

More recent analytic studies as well as simulations with massless SLAC fermions yield different results - they favor smaller values of $N_{\mathrm{r}}^{\text {crit }}$. Lattice models with these chiral fermions have the same internal symmetries as the continuum models. It was demonstrated that the $\mathrm{U}\left(2 N_{\mathrm{r}}\right)$ symmetry of the reducible model is never broken for any integer number of 4-component flavors [23]. In a subsequent publication the critical flavor number $N_{\mathrm{r}}^{\text {crit }}=$ $0.80(4)$ has been calculated [24]. Irreducible Thirring models with an odd number of flavors behave differently from the reducible models. They show a parity broken phase for $N_{\mathrm{f}} \lesssim 9$.

Independent simulations with 4-component domain-wall fermions (DWF), in which one adds an extra dimension to the Dirac operator, pointed to a critical flavor number $N_{\mathrm{r}}^{\text {crit }}$ below 2 [25]. In a follow-up publication with DWF, it was demonstrated that the model with $N_{\mathrm{r}}=1$ shows a phase transition with order parameter [26], implying that $1<N_{r}^{\text {crit }}<2$. The discrepancy of the results obtained with SLAC and DW fermions may be due to uncertainties in the extrapolation to an infinite domain-wall separation. In [26] it was speculated that the two lattice approaches describe different continuum theories, and that the bulk DWF formulation more closely conforms to a picture of the strong dynamics in which the auxiliary vector field resembles a gauge field.

\section{Lattice Simulations with Chiral SLAC Derivative}

In our simulations we use the chiral and non-local SLAC-derivative on a hypercubic lattice. The SLAC fermions have been used with great success to:

1. calculate the critical coupling of $\phi_{2}^{4}$-theory to high precision [27];

2. obtain an accurate value for the step scaling function in the two-dimensional nonlinear $\mathrm{O}(3)$ model;

3. calculate Ward-identities, the ground state structure, low-lying masses and the breaking or restoration of supersymmetry in low-dimensional supersymmetric WessZumino models [27,28];

4. find accurate values for the critical exponents of GN model [29,30];

5. discover inhomogeneous structures in the multi-flavor $\mathbb{Z}_{2}$ and $U(1)$ Gross-Neveu models [31,32].

Lattice models with SLAC fermions have various advantages. In the present context the most relevant one is that they inherit all global inner symmetries and discrete spacetimes symmetries of the continuum models. For example, the reducible lattice Thirring model in three dimensions is invariant under $\mathrm{U}\left(2 N_{\mathrm{r}}\right)$ chiral transformations and $\mathbb{Z}_{2}$-parity. The lattice derivative $\partial_{\mu}^{\text {slac }}$ is anti-hermitean such that $i \not \partial$ is hermitean. This property is used to prove that certain fermion operators have no sign problem. Furthermore, the auxiliary field $v_{\mu}$ in $\mathcal{D}_{m}$ is a non-compact site variable and not a link variable as in some other lattice formulations. A further advance is that the Dirac operator has no doublers, and no rooting is necessary to describe systems with a small flavor number. Last but not least SLAC lattice fermions are cheap compared to local chiral fermions.

It is well-known that the non-local SLAC derivative leads to problems in lattice gauge theories [33]. Indeed, when the ordinary lattice derivative $\partial$ is replaced by a covariant derivative $D$, then one can perform a local gauge transformation which does not change the action but sends the canonical momentum $p=\mathrm{i} \partial$ to the edge of the Briolloin zone, where $p$ jumps. In case a complete gauge fixing is available (because of the Gribov-problem such a fixing will not be continuous) the problem with the discontinuous dispersion relation may be overcome. This has been demonstrated in lower-dimensional supersymmetric gauge models [34]. 


\section{SLAC Derivative}

To find the SLAC derivative on a finite lattice one first Fourier-transforms a wave function, multiplies the transformed wave function with the momentum and transforms back to coordinate space. In the spatial directions, we impose periodic boundary conditions for which the momenta $p_{i}$ are from $2 \pi \mathbb{Z} / N_{s}$. We choose the $p_{i}$ symmetric to the origin such that the edge of the Brillouin zone has maximal distance from the pole of the propagator. As a result we need an odd number of momenta and hence an odd number of lattice points $N_{S}$ in the spatial directions:

$$
x \in\left\{x_{k} \mid k=1, \ldots, N_{s}\right\}, \quad x_{k}=\frac{2 \pi}{N_{s}}\left(k-\frac{N_{s}+1}{2}\right), \quad N_{s} \text { odd } .
$$

The anti-symmetric lattice derivative in a given spatial direction takes the form:

$$
\partial_{x x^{\prime}}^{\mathrm{p}}=\partial_{x-x^{\prime}}^{\mathrm{p}}= \begin{cases}0 & x=x^{\prime}, \\ \frac{\pi}{N_{s}} \frac{(-)^{k-k^{\prime}}}{\sin \left(x-x^{\prime}\right) / 2} & x \neq x^{\prime},\end{cases}
$$

where $x=x_{k}$ and $x^{\prime}=x_{k^{\prime}}$ are sites on the lattice defined in (32).

In the (imaginary) time direction the Fermi field fulfills anti-periodic boundary conditions. Then the momentum $p_{0}$ is from $2 \pi\left(\mathbb{Z}+\frac{1}{2}\right) / \beta$. To distribute the $p_{0}$ symmetric about the origin we need an even number of $p_{0}$ and thus an even number of lattice points in the time-direction,

$$
x \in\left\{x_{k} \mid k=1, \ldots, N_{t}\right\}, \quad x_{k}=\frac{2 \pi}{N_{t}}\left(k-\frac{N_{t}}{2}\right), \quad N_{t} \text { even. }
$$

In this case, the lattice derivative reads:

$$
\partial_{x x^{\prime}}^{\mathrm{ap}}=\partial_{x-x^{\prime}}^{\mathrm{ap}}= \begin{cases}\frac{\mathrm{i} \pi}{L} & x=x^{\prime}, \\ \frac{\pi}{N_{t}} \frac{(-)^{k-k^{\prime}}}{\sin \left(x-x^{\prime}\right) / 2} e^{\mathrm{i}\left(x-x^{\prime}\right) / 2} & x \neq x^{\prime} .\end{cases}
$$

For finite-temperature systems in 2 spatial dimensions the SLAC derivatives $\partial_{\mu}^{\text {slac }}$ take the form:

$$
\partial_{0, \xi^{\prime}}^{\text {slac }}=\partial_{\tilde{\xi}_{0}}^{\mathrm{ap}} \delta_{\tilde{\xi}_{1}} \delta_{\xi_{2}}, \quad \partial_{1, \xi^{\prime}}^{\text {slac }}=\delta_{\xi_{0}} \partial_{\xi_{1}}^{\mathrm{p}} \delta_{\tilde{\xi}_{2}}, \quad \partial_{2, \xi}^{\text {slac }}=\delta_{\xi_{0}} \delta_{\xi_{1}} \partial_{\tilde{\xi}_{2}}^{\mathrm{p}},
$$

where we abbreviated $\delta_{\xi_{1}} \equiv \delta_{0 \xi_{1}}$.

Below, we shall present the results of simulations at low temperature on a $N \times(N-1)^{2}$ lattices with $N=8,12,16,24$. In the simulations we used pseudo-fermions and the rational HMC-algorithm with operator:

$$
\left(\operatorname{det}\left(\mathcal{D}_{0}^{\dagger} \mathcal{D}_{0}\right)^{N_{\mathrm{f}} / 2 N_{\mathrm{PF}}}\right)^{N_{\mathrm{PF}}}, \quad N_{\mathrm{PF}} \approx 2 N_{\mathrm{f}} .
$$

The inverse of the shifted operator enters the rational approximation based on a multimass conjugate gradient (CG) solver. During the CG iterations the derivative is applied many times to a pseudo-fermion field. Thereby we make use of a special property of the SLAC derivative: It is diagonal in momentum space, such that

$$
\left(\mathcal{D}_{m} \psi\right)(x)=\mathcal{F}^{-1}[\mathrm{i} p \mathcal{F}[\psi](p)](x)+\left(\mathrm{i} \gamma^{\mu} v_{\mu}(x)+m\right) \psi(x),
$$

where $\mathcal{F}$ denotes the Fourier transformation. Instead of using a three-dimensional (parallelized) Fourier transformation, we apply one-dimensional Fourier transformations that are computed in parallel.

To estimate fermion propagators we used $N_{\text {est }} \approx 200 N_{\mathrm{f}}$ stochastic estimators. For most measurements we generated approximately 5000 configurations. We estimated the finite size corrections and checked that they are under control. 
For the models with $N_{\mathrm{r}} \in[0.5,1.1]$ we used the parity-even extensions to non-integer $N_{\mathrm{r}}$. More precisely, after integrating out the fermions we arrive at the effective action (26) in which $N_{\mathrm{r}}$ is only a prefactor. We then continue $N_{\mathrm{r}} \in \mathbb{N}$ to real values. This formal procedure is similar to other studies where $N_{\mathrm{r}}$ only appears as a parameter, but we should stress that for $N_{\mathrm{r}} \notin \mathrm{N}$ such a definition most likely does not describe a local quantum field theory in the continuum. In particular, reducible models with half-integer $N_{\mathrm{r}}$ obtained via this procedure need not be equivalent to irreducible models with integer $N_{\mathrm{f}}=2 N_{\mathrm{r}}$.

\section{Dual Formulation and Effective Potentials}

Similarly as in the fermion bag algorithm of Chandrasekharan [35], one can integrate out the interaction part of the partition sum to obtain a formulation in terms of new spin like variables $k_{x i}^{a b} \in\{0,1\}$, where $i$ relates to the spinor degree of freedom and $a, b$ to the flavor degree of freedom $[29,36]$. For non-local SLAC-fermions the dual formulation is a bit more involved than for ultra-local staggered fermions. Actually, it is advantageous to use the Fierz-identity (19) and bosonize the resulting four-Fermi theory. For $m=0$ this yields the tensor-scalar formulation:

$$
\mathcal{L}=\bar{\Psi}(\mathrm{i} \not \partial+\mathrm{i} T+\mathrm{i} \phi) \Psi+\frac{N_{\mathrm{f}}}{4 g^{2}} \operatorname{tr} T^{2}+\frac{N_{\mathrm{f}}}{2 g^{2}} \phi^{2}, \quad T^{\dagger}=T, \phi \in \mathbb{R},
$$

which is equivalent to the vector formulation of the Thirring model. Differentiating the Boltzmann factor with respect to the components of the tensor field $T_{a b}$ yields the DysonSchwinger equations:

$$
\left\langle T_{a b}\right\rangle \propto\left\langle\bar{\psi}_{a} \psi_{b}\right\rangle .
$$

Since $T$ transforms under chiral rotations as $T \rightarrow U T U^{\dagger}$, the expectation value $\left\langle T_{a b}\right\rangle$ serves as an order parameter for chiral symmetry. In the scalar-tensor formulation we can probe for condensates in all channels represented by $T_{a b}$. For example a parity breaking condensate, a chirality breaking condensate or a non-time-reversal invariant Haldaneterm [37]. Unfortunately, this formulation has a severe sign problem and cannot be used directly in simulations. But exploiting its dual formulation we are able to express various quantities of interest, for example, coefficients in the expansion of the effective potential, to expectation values in the sign-problem free vector formulation.

\subsection{Effective Potential versus Condensates}

The expectation value $\left\langle T_{a b}\right\rangle$ can be diagonalized by a chiral rotation and it suffices to calculate the effective potential (denoted by $V$ to distinguish it from the effective potential in the vector formulation) on diagonal order parameters $\langle T\rangle=t_{i} H^{i}$, where $\left\{H^{i}\right\}$ forms a basis of the space of diagonal hermitean matrices,

$$
V\left(\left\langle T_{a b}\right\rangle\right)=V(t)=-\frac{1}{V} \log \sum_{n=0}^{2 N_{\mathrm{f}}} \sum_{i=1}^{N_{\mathrm{f}}} a_{n, i}\left(t_{i}\right)^{n} .
$$

with the help of the dual formulation one proves that the coefficients $a_{n, i}$ are given by expectation values of powers of fermion bilinears and these expectation values can be calculated in the vector formulation.

The minima of $V$ are attained for fields:

$$
\langle T\rangle_{\min }=\frac{2 x}{N_{\mathrm{f}}} \operatorname{diag}(\underbrace{1, \ldots, 1}_{n_{+}}, \underbrace{-1, \ldots,-1}_{n_{-}}), \quad n_{+} \geq n_{-},
$$

where permutations of the diagonal elements lead to equivalent minima. The physically distinct ones are characterized by the amplitude $x$ and by

$$
n=n_{+}-n_{-}= \begin{cases}0,2,4, \ldots, N_{\mathrm{f}}, & \text { for } N_{\mathrm{f}} \text { even } \\ 1,3,5, \ldots, N_{\mathrm{f}} & \text { for } N_{\mathrm{f}}, \text { odd }\end{cases}
$$


An order parameter (42) with $x>0$ gives rise to the symmetry breaking pattern

$$
U\left(N_{\mathrm{f}}\right) \otimes \mathbb{Z}_{2} \rightarrow U\left(n_{+}\right) \otimes U\left(n_{-}\right),
$$

which is different for an odd and an even number of irreducible flavors. For example, for three and four flavors we find:

$$
\begin{array}{ll}
N_{\mathrm{f}}=4: & \left(n, n_{+}, n_{-}\right) \in\{(0,2,2),(2,3,1),(4,4,0)\}, \\
N_{\mathrm{f}}=5: & \left(n, n_{+}, n_{-}\right) \in\{(1,3,2),(3,4,1),(5,5,0)\} .
\end{array}
$$

Only reducible models with even $N_{\mathrm{f}}=2 N_{\mathrm{r}}$ permit a symmetry breaking with $n=0$, in which case $\mathrm{U}\left(2 N_{\mathrm{r}}\right)$ breaks to $\mathrm{U}\left(N_{\mathrm{r}}\right) \times \mathrm{U}\left(N_{\mathrm{r}}\right)$. This breaking is induced by a staggered condensate $\sum(-1)^{a} \bar{\psi}_{a} \psi_{a}$, which corresponds to a chiral condensate $\bar{\Psi} \Psi$ (and not the Haldane mass) in the reducible formulation, see Equation (25). In this channel the broken system is parity invariant. In Figure 1 , the potentials for $N_{\mathrm{f}}=4$ and the three feasible breaking patterns in (45) are depicted. The left panel shows the analytic results in the strong coupling limit whereas the right panel shows the results of simulations on a $16 \times 15 \times 15$-lattice with inverse coupling $\lambda=N_{\mathrm{f}} / 2 g^{2}=0.118$.
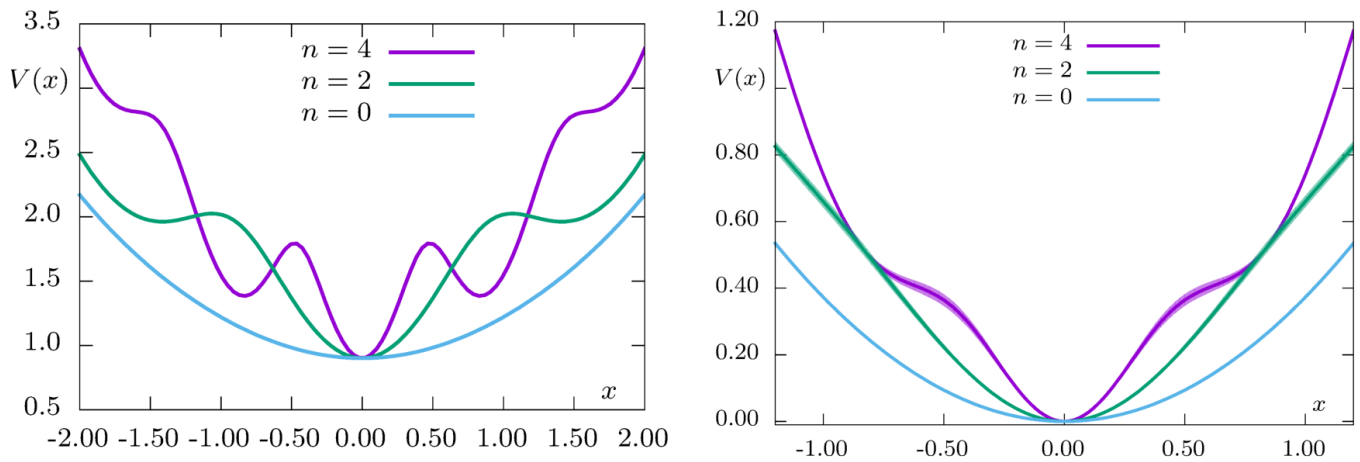

Figure 1. Effective potential for the three channels of the Thirring model with $N_{\mathrm{r}}=2$ or equivalently $N_{\mathrm{f}}=4$ flavors. Left panel: strong coupling limit. Right panel: simulation on a lattice with $N=16$ and inverse coupling $\lambda=0.118$. Figures taken from [23].

We see that in all three channels the minimum of the effective potential is at $\langle T\rangle=0$. Thus, we do not observe spontaneous symmetry breaking (SSB) for the given $\lambda$ and on the chosen lattice. With increasing inverse coupling $\lambda$ the minima at the origin get more pronounced and the only way to see SSB is to decrease $\lambda$. We shall see below, that for all admitted values of $\lambda$, we see no SSB in the reducible models.

For an odd number of irreducible flavors the situation looks differently. Figure 2 shows the effective potentials in the three channels (46) available for $N_{\mathrm{f}}=5$.
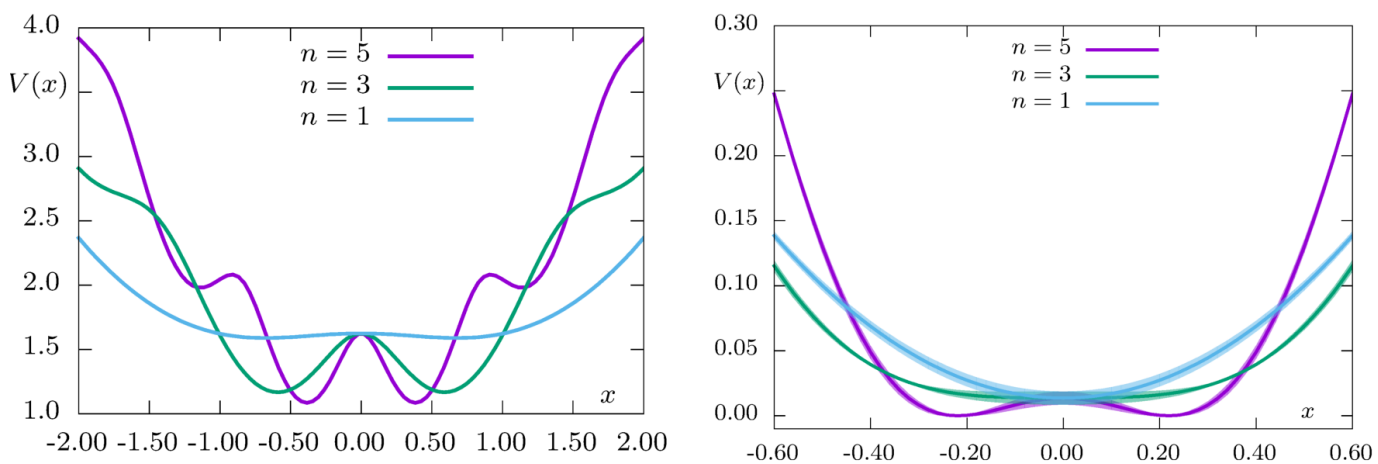

Figure 2. Effective potential for the three channels of the Thirring model with $N_{\mathrm{f}}=5$ irreducible flavors. Left panel: strong coupling limit. Right panel: simulation on a lattice with $N=16$ and inverse coupling $\lambda=0.102$. Figures taken from [23]. 
Again, the result in the strong coupling limit is shown in the left panel and the simulation results for $\lambda=0.102$ in the right panel. We observe that a condensate $\langle T\rangle$ with $n=5$ forms. This condensate does not break the chiral symmetry $\mathrm{U}(5)$ but it breaks parity. If one decreases the coupling $g^{2}$ then one observes a transition into the symmetric phase.

An interesting observable in this context is the lattice filling factor:

$$
k=\frac{1}{2 V N_{\mathrm{f}}} \sum_{i=1}^{2} \sum_{a, b=1}^{N_{\mathrm{f}}} \sum_{x=1}^{V} k_{x i}^{a b} \in[0,1],
$$

which relates to the number of states occupied by the interaction on a lattice site-a natural variable in the dual formulation. It counts how many fermions take part in the non-trivial interaction. In the weak coupling limit $\lambda \rightarrow \infty$, the filling factor vanishes and in the strong coupling limit $\lambda \rightarrow 0$ it is one. It cannot exceed the value $k=1$ because of Pauli-blocking on every site. On the left in Figure 3, we depicted the average lattice filling factor, given by a 4-Fermi correlation function,

$$
\langle k\rangle=-\frac{1}{2 N_{\mathrm{f}}} \frac{\lambda}{V} \frac{\dot{Z}(\lambda)}{Z(\lambda)}+c=\frac{1}{4 N_{\mathrm{f}} \lambda}\left\langle j^{\mu} j_{\mu}\right\rangle+c,
$$

for Thirring models with flavor number between 2 and 11 and lattice sizes $N=8,12$ and 16 . We see clearly that at strong couplings $\lambda \lesssim \lambda^{*}$, the systems are in a lattice-artifact phase dominated by Pauli blocking. We also see that the average filling factor does not suffer much from finite size corrections, in particular in the strong coupling region.
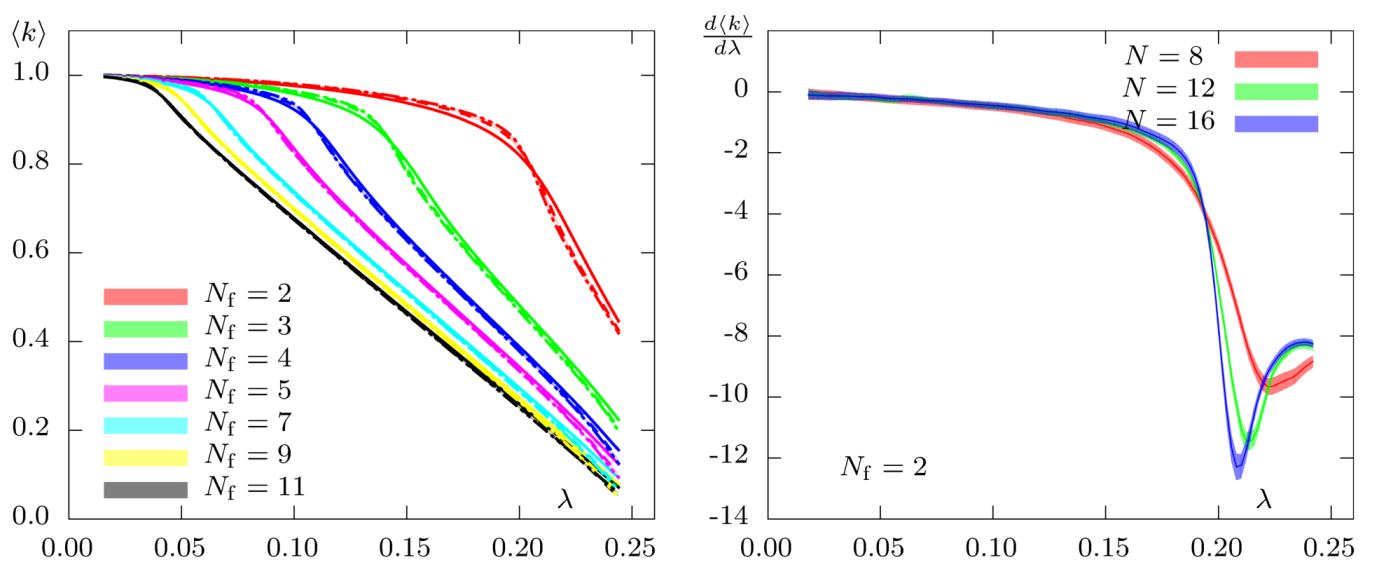

Figure 3. Left: average lattice filling factor (48) on three lattices for every flavor between 2 and 11. Right: With increasing lattice size a singularity of the susceptibility (49) builds up, here for 2 irreducible flavors. Figures taken from [23].

The susceptibility associated with $k$ is given by a 8-Fermi correlation function,

$$
\partial_{\lambda}\langle k\rangle=-\frac{1}{16 N_{\mathrm{f}} \lambda^{3}} \sum_{x}\left\langle\left(j^{\mu} j_{\mu}\right)(x)\left(j^{\mu} j_{\mu}\right)(0)\right\rangle_{c}+\frac{\mathrm{c}-\langle k\rangle}{\lambda},
$$

and its dependence on $\lambda$ is seen in the right panel of Figure 3 for the model with $N_{\mathrm{f}}=2$ on three different lattice sizes. The dip of the susceptibility increases with the lattice size and this points to a transition from a strong-coupling lattice artifact phase to a phase, which connects to continuum physics.

\subsection{Spontaneous Symmetry Breaking of Parity for Odd $N_{\mathrm{f}} \leq N_{\mathrm{f}}^{\text {crit }}$}

For odd $N_{\mathrm{f}}$ the approximate critical coupling $\lambda^{*}$ on lattices with $N=8,12$ and 16 are listed in Table 2. 
Table 2. The critical couplings $\lambda^{*}$ separating the artifact phase and physical phase for three lattice sizes and the Thirring lattice models with an odd number of flavors.

\begin{tabular}{cccccc}
\hline $\boldsymbol{N}$ & $\boldsymbol{N}_{\mathbf{f}}=\mathbf{3}$ & $\mathbf{5}$ & $\mathbf{7}$ & $\mathbf{9}$ & $\mathbf{1 1}$ \\
\hline 8 & $\lambda^{*}=0.158(4)$ & $0.098(2)$ & $0.073(2)$ & $0.058(2)$ & $0.048(2)$ \\
12 & $\lambda^{*}=0.149(4)$ & $0.094(3)$ & $0.068(2)$ & $0.054(2)$ & $0.046(2)$ \\
16 & $\lambda^{*}=0.146(4)$ & $0.091(2)$ & $0.068(2)$ & $0.054(2)$ & $0.046(2)$ \\
\hline
\end{tabular}

A critical coupling $\lambda_{c}$ of a transition, where a condensate forms, must be larger than $\lambda^{*}$ to stay away from the artifact phase. We have seen earlier that if a condensate forms, then it is the parity-breaking condensate with $n=N_{\mathrm{f}}$. This means that for odd $N_{\mathrm{f}}$ the system is either in the symmetric phase or develops a condensate, which does not break chiral symmetry but only parity. Figure 4 shows the curvature $\kappa$ of the effective potential in the direction with $n=N_{\mathrm{f}}$ as a function of the inverse coupling. The bars show the estimated infinite volume extrapolation of $\lambda^{*}$, such that only values to the right of these bars are in the phase which connects to continuum physics. For example, for $N_{\mathrm{f}}=3$ the effective potential becomes unstable against condensation of $\left\langle\bar{\psi}_{a} \psi_{b}\right\rangle \propto \delta_{a b}$ at the inverse coupling $\lambda_{c} \approx 0.172$, well outside of the lattice artifact phase at strong couplings $\lambda \lesssim \lambda^{*} \approx 0.145$.

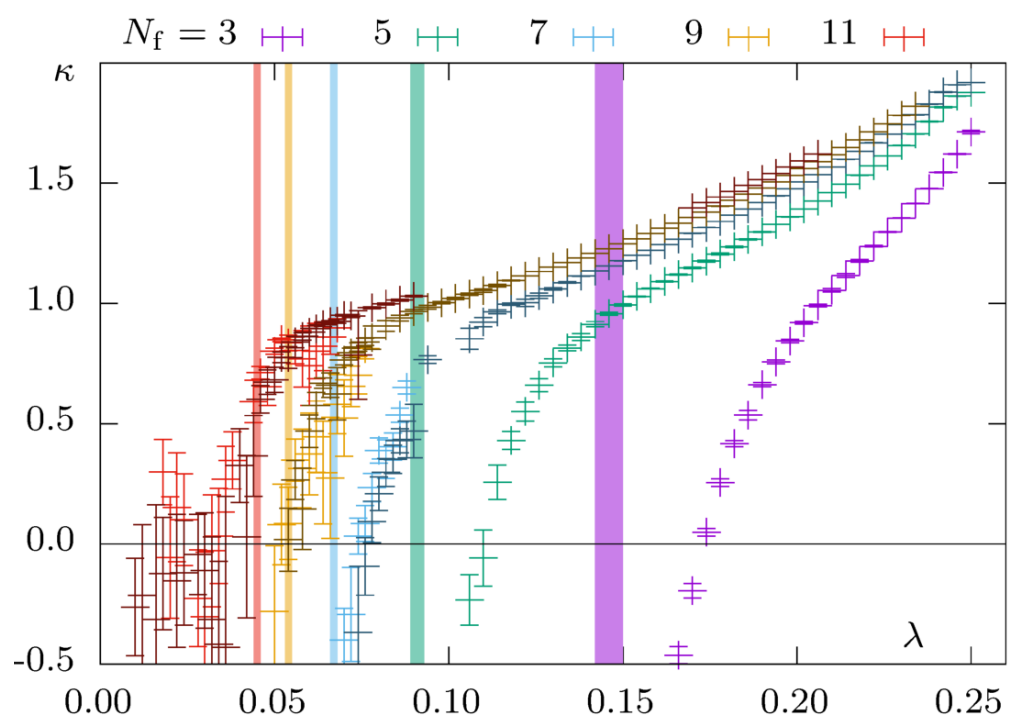

Figure 4. The curvature $\kappa$ of the effective potentials at the origin is shown for odd $N_{\mathrm{f}}$. The curvature is taken in the channel with $n=0$, since in the other channels the curvature is larger. Figure taken from [23].

We observe a similar instability against condensation for all odd flavor numbers between $1 \leq N_{\mathrm{f}} \leq 9$. Actually, we are not sure that parity is broken for $N_{\mathrm{f}}=9$, since the critical couplings $\lambda^{*}=0.51$ and $\lambda_{c}=0.53$, measured on a lattice with $N=20$, are almost identical. But our simulations clearly reveal that there is no broken phase for $N_{\mathrm{f}} \geq 11$ and there is a broken phase for $N_{\mathrm{f}} \leq 7$. The critical flavor number $N_{\mathrm{f}}^{\text {crit }} \approx 9$ for two-component flavors corresponds to a critical flavor number $N_{\mathrm{r}}^{\text {crit }} \approx 4.5$ for four-component flavors and thus is in good agreement with some of the earlier results in Table 1.

\subsection{Special Case: $N_{\mathrm{f}}=1$}

While the models with even $N_{\mathrm{f}}$ have no sign problem, we found numerically that a possible sign problem for odd $N_{\mathrm{f}}$ is extremely mild (not observed in our ensembles) for all $N_{\mathrm{f}} \geq 3$. Only the irreducible single-flavor Thirring model has a severe sign problem that hinders a direct simulation. 
In order to still perform simulations, we formulated a fermion-bag-like algorithm: Starting from the bosonized Fierz-transformed formulation (22), one expands the Boltzmann factor in powers of the Yukawa interaction,

$$
Z=\int \mathcal{D} \sigma \mathcal{D} \psi \mathcal{D} \bar{\psi} e^{-S_{0}[\psi]-\lambda \sum \sigma_{x}^{2}} \prod_{x, i} \sum_{k_{x i}=0}^{1} \frac{\left(-\sigma_{x} \bar{\psi}_{x}^{i} \psi_{x}^{i}\right)^{k_{x i}}}{k_{x i} !},
$$

where $S_{0}$ is the lattice action of free SLAC fermions and for simplicity we already set $m=0$. The coefficient $\lambda=1 / 6 g^{2}$ of $\sigma^{2}$ is different from the coefficient $\lambda$ of $v_{\mu} v^{\mu}$ in Sections 5.1 and 5.2. The product and sum combine into one large sum over sets of occupation number variables $k_{x i}$ which only assume the values 0 and 1 due to the Grassmann nature of $\psi$ and $\bar{\psi}$. Now, one can see that whenever $k_{x i}=1$ the Berezin integrals over $\psi_{x}^{i}$ and $\bar{\psi}_{x}^{i}$ are saturated by the Yukawa term. The kinetic term must, hence, contribute trivially which is equivalent to removing the rows and columns $(x, i)$ from $\not$. The remaining fermionic and bosonic integrals decouple leaving a fermion determinant and a bosonic weight factor,

$$
Z=\sum_{\left\{k_{x}\right\}}(-1)^{k V} w(k) \operatorname{det} \mathrm{i} \not\left[\left\{k_{x}\right\}\right],
$$

where $\not\left[\left\{k_{x}\right\}\right]$ denotes the reduced fermion operator, the lattice filling factor $k$ has been defined in (47) and

$$
w(k)=\left(\sqrt{\frac{\lambda}{\pi}} \int \mathrm{d} \sigma \sigma^{2} e^{-\lambda \sigma^{2}}\right)^{k V} .
$$

One should note that the bosonic integral over $\sigma_{x}$ vanishes whenever $k_{x 1} \neq k_{x 2}$. Thus, we used the notation $k_{x} \equiv k_{x 1}=k_{x 2}$. This result is similar to the fermion bag algorithm [35] with the notable difference that we do not find "bags" in the sense of connected clusters due to the non-local nature of chiral SLAC fermions. (A much more detailed derivation of these formulae can be found in [36])

In order to generate the Markov chain, we use simple Metropolis updates starting from the completely filled lattice with $\operatorname{det} i \not[\{1, \ldots, 1\}]=1$. However, we found that flipping a single $k_{x}$ often yields configurations of vanishing weight such that we also propose simultaneous flips of two $k_{x}$. To speed up the updates of the fermion determinant, we employ a combination of matrix determinant lemmas and the Sherman-MorrisonWoodbury formula.

While we can prove that detið[\{k $\left.\left.k_{x}\right\}\right]$ is real and positive [36], the sign factor $(-1)^{k V}$ remains problematic. However, we found numerically that

$$
\langle k\rangle=\frac{1}{Z} \sum_{\left\{k_{x}\right\}} k(-1)^{k V} w(k) \operatorname{det} i \not\left[\left\{k_{x}\right\}\right]
$$

does not suffer the usual increase in variance and instead yields a reliable signal. This expectation value enters the expression for the local effective potential $V_{\text {loc }}$. The latter characterized the distribution function $\exp \left(-V_{\mathrm{loc}}\right)$ of $\sigma_{x}$ and yields the partition function,

$$
Z=\int \mathrm{d} \sigma_{x} e^{-V_{\mathrm{loc}}\left(\sigma_{x}\right)}
$$

for an arbitrary $x$. Due to translational symmetry we assume that the distribution of $\sigma_{x}$ is independent of $x$. The potential has the form:

$$
V_{\mathrm{loc}}\left(\sigma_{x}\right)=\lambda \sigma_{x}^{2}+\ln \left(a_{0}+a_{2} \sigma_{x}^{2}\right),
$$


wherein the coefficients are related in a straightforward way to expectation values of moments of $k$. One should stress that $V_{\mathrm{loc}}$ is not quite the standard constraint effective potential. However, as it describes the statistics of the local order parameter, we expect it to accurately describe the physics of the system.

Using the (positive) position of the minimum of the local effective potential as an order parameter, a detailed study of the system was performed in [36]. Here, we collect the main results: The critical coupling was found to be:

$$
\lambda_{c}= \begin{cases}0.3804(3) & \text { from the condensate, } \\ 0.3813(3) & \text { from the susceptibility. }\end{cases}
$$

The condensate and susceptibility critical exponents are given as:

$$
\beta=0.406(8), \quad \gamma=1.1(3) .
$$

The transition to the lattice artifact phase was also analyzed. It occurred at

$$
\lambda^{*}=0.32838(9)
$$

There, the data were consistent with a second order phase transition which is different from the behavior of even flavors. However, one should note that its equivalence to the single-flavor GN model might render the $N_{\mathrm{f}}=1$ Thirring model a special case.

\subsection{No Spontaneous Symmetry Breaking for Any Even $N_{\mathrm{f}}$ or $N_{\mathrm{r}} \geq 1$}

For Thirring models with even $N_{\mathrm{f}}$-these are equivalent to reducible models with $N_{\mathrm{r}}=\frac{1}{2} N_{\mathrm{f}}$ - the situation is quite different. Figure 5 shows the curvature $\mathcal{K}$ of the effective potential $V$ at the origin in the different channels for 2 and 4 irreducible flavors. We see that for all $\lambda \geq \lambda^{*}$ the curvatures are positive such that no condensate can form in the phase, which connects to continuum physics. This striking result will be further substantiated in the following sections.
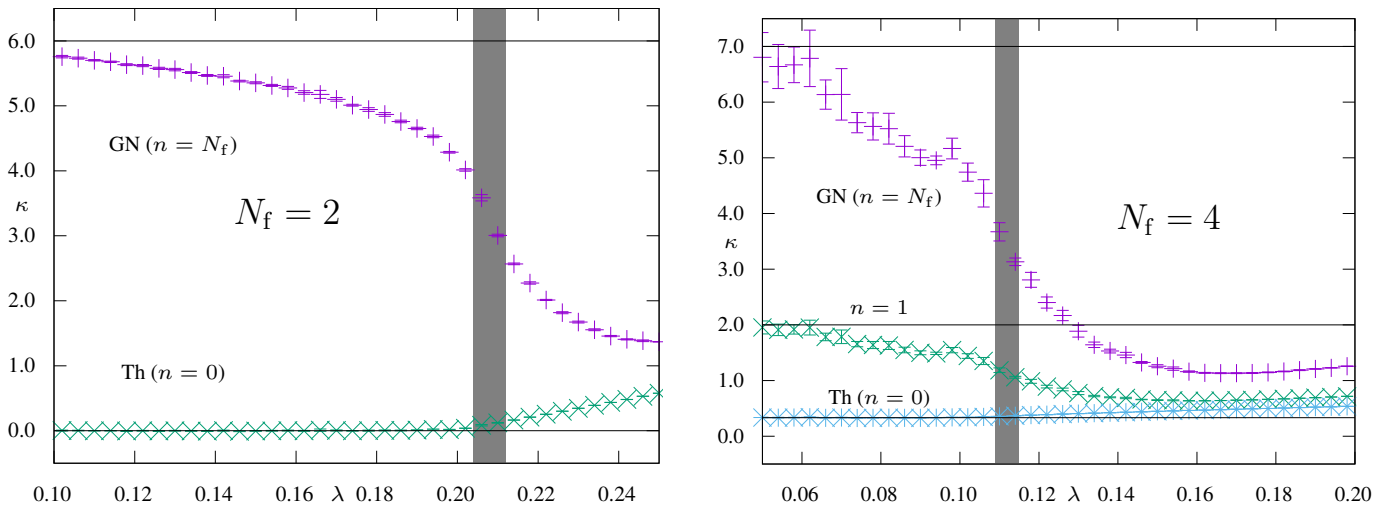

Figure 5. The curvatures $\kappa$ of the effective potentials in the channels labelled by the integer $n$ defined in (43). Left: Thirring model with $N_{\mathrm{f}}=2$. Right: Thirring model with $N_{\mathrm{f}}=4$.

\section{Banks-Casher Relations}

The effective action of the massive reducible Thirring models with $N_{\mathrm{f}}=2 N_{\mathrm{r}}$ reads:

$$
S_{\text {eff }}=\frac{1}{2 g^{2}} \int \mathrm{d}^{3} x v^{\mu} v_{\mu}-\ln \operatorname{det}\left(m^{2}-\mathcal{D}_{0}^{2}\right),
$$


where $\mathcal{D}_{0}$ has been introduced below (23). It is used to investigate the parity-invariant condensate of the reducible models in the channel with $n=0$, in which the symmetry $\mathrm{U}\left(2 N_{\mathrm{r}}\right)$ breaks to $\mathrm{U}\left(N_{\mathrm{r}}\right) \times \mathrm{U}\left(N_{\mathrm{r}}\right)$ and in which the effective potential has minimal curvature. The parity-even condensate is:

$$
\begin{aligned}
\Sigma & =\frac{1}{V} \frac{1}{Z} \int \mathcal{D} v^{\mu} \operatorname{tr}\left(\frac{m}{m^{2}-\mathcal{D}_{0}^{2}}\right) e^{-N_{\mathrm{f}} S_{\mathrm{eff}}(v)} \\
& =\frac{2 m}{V} \int_{0}^{\infty} \frac{\mathrm{d} E}{m^{2}+E^{2}} \bar{\rho}(E)
\end{aligned}
$$

and it contains the average spectral density $\bar{\rho}$, defined by:

$$
\bar{\rho}(E)=\frac{1}{Z} \int \mathcal{D} v^{\mu} e^{-N_{\mathrm{f}} S_{\text {eff }}(v)} \rho_{v}(E) .
$$

The spectral density of the Dirac operator $\mathrm{i} \mathcal{D}_{0}$ for a fixed $v_{\mu}$ is defined by

$$
\operatorname{tr} f\left(\mathrm{i} \mathcal{D}_{0}\right)=\int_{-\infty}^{\infty} \mathrm{d} E f(E) \rho_{v}(E) \text {. }
$$

The relation (60) implies that the condensate $\Sigma$ vanishes for $m \rightarrow 0$ in case the integral over $E$ is finite. A condensate forms for small $m$ if the integral is proportional to $1 / m$. This happens when for a typical configuration there is an abundance of low-lying eigenvalues of $-\mathcal{D}_{0}^{2}$. This is the content of the celebrated Banks-Casher relation which states that in the infinite volume limit the condensate is proportional to $\bar{\rho}(0)$. Figure 6 shows the average spectral densities for $N_{\mathrm{r}}=1$ (left panel) and for $N_{\mathrm{r}}=0.8$ (right panel).
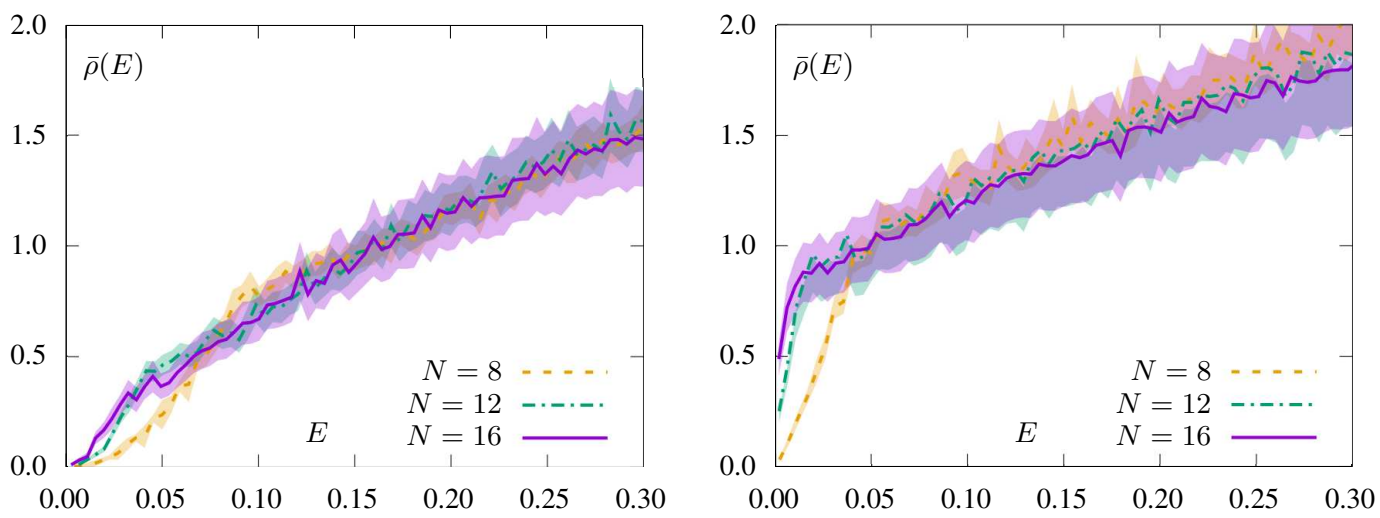

Figure 6. The average spectral density $\bar{\rho}$ of the Dirac operator for 1 reducible flavor (left panel) and for 0.8 reducible flavors (right panel) [24].

While small eigenvalues accumulate for the smaller flavor number, this does not happen for one reducible flavor. This indicates that there is no spontaneous breaking of chiral symmetry in all reducible Thirring models with $N_{\mathrm{r}} \geq 1$, in contrast to many previous studies. It also shows that there is a condensation of small eigenvalues for $N_{\mathrm{r}}=0.8$.

The next step beyond using the Banks-Casher relation could be to exploit the (for non-Abelian gauge theories) well-established link between chiral properties and chiral random matrix theory (cRMT) which describes universal statistical properties of low-lying eigenvalues of the Dirac operator [38]. The constraints imposed by chiral symmetry and its spontaneous breaking determine the structure of low-energy effective partition function. The RMT allows for a determination of the chiral condensate from simulations at finite volume (and nonzero lattice constant $a$ ). For SLAC fermions, the lattice Dirac operator for SLAC fermions is hermitean and in a possible broken phase the statistical properties of the low-lying Dirac eigenvalues would be determined by the chiral condensate. In future studies it should be interesting to compare the distribution of individual eigenvalues 
or the spacing between them to RMT prediction to arrive at more stable infinite volume extrapolations-both for the breaking of parity in the irreducible models and the breaking of 'chirality' in the reducible ones.

\section{Spectrum of Low-Lying States}

If the $\mathrm{U}\left(2 N_{\mathrm{r}}\right)$-symmetry was spontaneously broken, the particle spectrum would reveal the existence of Goldstone modes. In formulations with domain wall fermions the analysis of the spectrum indicates SSB for $N_{\mathrm{r}}=1$. This is in conflict with the results obtained with the effective potential and spectral density for chiral SLAC fermions, which clearly show that $N_{\mathrm{r}}^{\text {crit }}<1$.

To clarify the situations for $N_{\mathrm{r}} \approx 1$ we study the spectrum of light mesons. The four interpolating operators are:

$$
\mathcal{O}_{a}(x)=\bar{\psi}(x)\left(\sigma_{a} \otimes \sigma_{0}\right) \psi(x), \quad \mathcal{O}_{a}(t)=\sum_{x} \mathcal{O}_{a}(t, x), \quad 0 \leq a \leq 3,
$$

where $\sigma_{0}=\mathbb{1}_{2}$ and $\sigma_{1}, \sigma_{2}, \sigma_{3}$ are the Pauli-matrices and we use the reducible formulation (23) of the Thirring models. Then the expectation values of $\mathcal{O}_{3}$ and $\mathcal{O}_{0}$ are identified as chiral and parity condensates. The latter vanishes in the parity-invariant reducible models. The correlation matrix of the interpolating operators is diagonal, $C_{a b}(t)=\left\langle\mathcal{O}_{a}(t) \mathcal{O}_{b}(t)\right\rangle_{c}=C_{a}(t) \delta_{a b}$, and is used to extract the masses of the light mesons. One can show that the $C_{1}$ and $C_{2}$ are equal. If the symmetry $\mathrm{U}(2)$ is not broken, then we should see a singlet and triplet of $\mathrm{U}(2)$ in the spectrum. The correlation functions $C_{1}, C_{2}, C_{3}$ belong to this triplet. On the other hand, if $U(2)$ is spontaneously broken to $U(1) \otimes U(1)$, then we should detect two Goldstone bosons, related to the interpolating operators $\mathcal{O}_{1}$ and $\mathcal{O}_{2}$.

From the correlation functions on a relatively small lattice with $N=12$ and a larger lattice with $N=16$ (see Figure 7) we extracted the masses given in Table 3. We see that for $N_{\mathrm{r}}=1.0$ the masses extracted from $C_{1,2}$ and $C_{3}$ are comparable. They belong to the expected triplet in the symmetric phase. On the other hand, for $N_{\mathrm{f}}=0.8$ we do not see such a triplet. Only $m_{1}$ and $m_{2}$ are degenerate and they are identified as Goldstone particles. In the broken phase the correlation function $C_{0}$ falls off rapidly and we cannot extract a reliable value for the mass on the relatively small lattices considered. Hence this mass is missing in the broken phase in the Table.
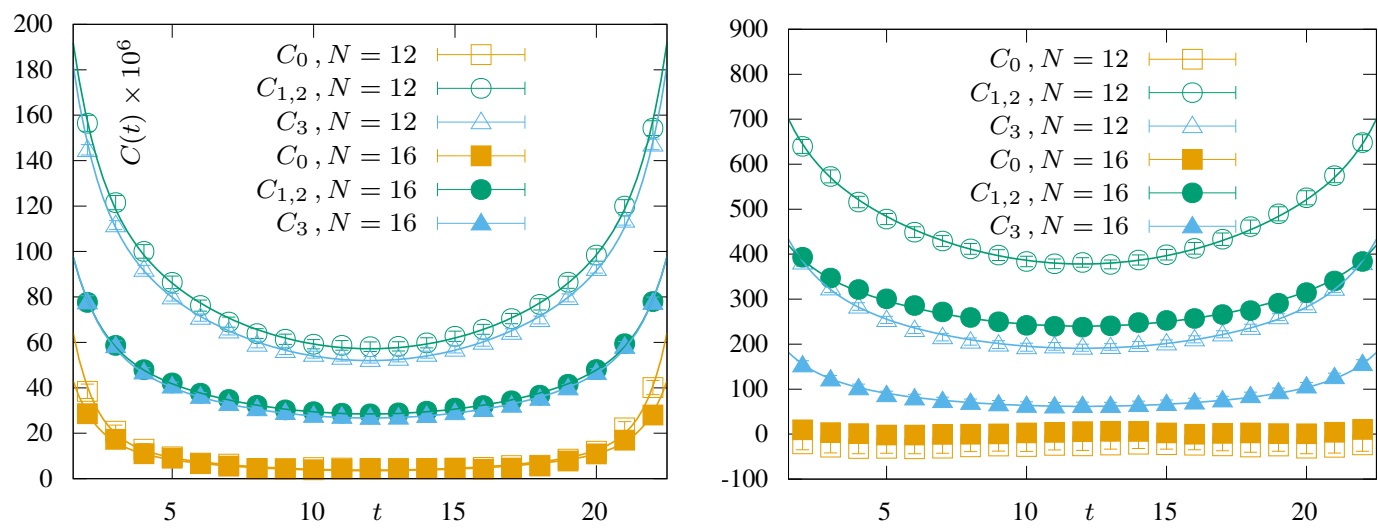

Figure 7. The correlation functions $C_{0}, C_{1}, C_{2}$ and $C_{3}$ of the interpolating operators for the low-lying mesons on lattice of various sizes. On the left for $N_{\mathrm{r}}=1$ and on the right for $N_{\mathrm{r}}=0.8$ [24]. 
Table 3. Masses of the lightest mesons on two lattices for $N_{\mathrm{r}}=1.0$ and $N_{\mathrm{r}}=0.8$ reducible flavors.

\begin{tabular}{ccccc}
\hline$C$ & $m(\mathbf{1 2})$ & $m(\mathbf{1 6})$ & $N_{\mathbf{r}}$ & Symm. \\
\hline$C_{0}$ & $0.21(2)$ & $0.21(2)$ & 1.0 & \\
$C_{1,2}$ & $0.134(3)$ & $0.128(2)$ & 1.0 & $\mathrm{U}(2)$ \\
$C_{3}$ & $0.138(2)$ & $0.131(2)$ & 1.0 & \\
\hline$C_{1,2}$ & $0.103(2)$ & $0.095(3)$ & 0.8 & $\mathrm{U}(1) \times \mathrm{U}(1)$ \\
$C_{3}$ & $0.109(4)$ & $0.127(7)$ & 0.8 & \\
\hline
\end{tabular}

As earlier, we conclude that the critical flavor number $N_{\mathrm{r}}^{\text {crit }}$ is smaller than 1 . We also conclude that it is larger than 0.8 .

\section{Estimating the Critical Flavor Number $N_{\mathrm{r}}^{\text {crit }}$}

To extract a reliable estimate for $N_{\mathrm{r}}^{\text {crit }}$ we performed a detailed finite size analysis of the susceptibility, $\partial_{\lambda}\langle k\rangle$ in (49) on a grid of $N_{\mathrm{r}}$-values between 0.5 and 1.0. The simulation results reveal two dips of the susceptibility for all $N_{\mathrm{r}} \gtrsim 0.78$. Three examples are depicted in Figure 8, left panel. The dips become more pronounced with increasing system size, as seen in the same Figure on the right. The dip at stronger coupling belongs to the transition into the lattice artifact phase discussed above. More interestingly, we see a second dip at weaker coupling (larger $\lambda$ ). Most likely, it points to a phase transition without order parameter.
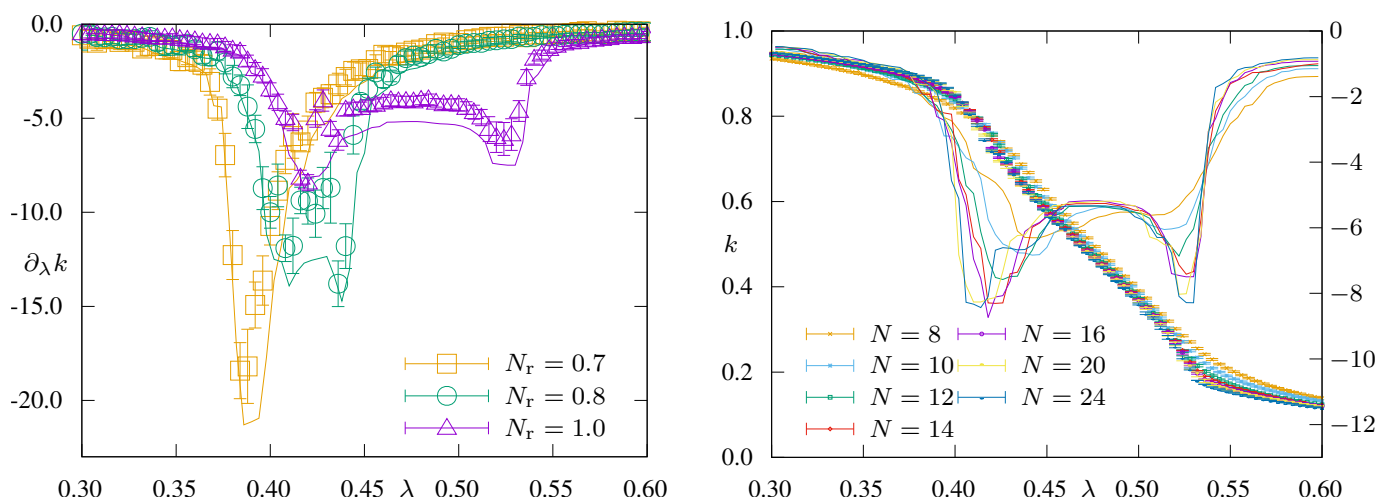

Figure 8. The susceptibility (49) for Thirring models with $N_{\mathrm{r}}=0.7,0.8$ and corresponding susceptibility for $N_{\mathrm{r}}=1.0$ reducible flavors (left panel) and the volume-dependence of the the average filling factor (48) and corresponding susceptibility (right panel).

The positions of the susceptibility dips on a fine grid in the $\left(\lambda, N_{\mathrm{r}}\right)$-plane near $N_{\mathrm{r}} \approx 1$ are calculated with an expensive scan of the susceptibility as function of the inverse coupling $\lambda$. The resulting phase transition lines of the (probably first order) ubiquitous lattice artifact transitions and of the (probably smooth) new transitions for all $N_{\mathrm{r}} \gtrsim 0.78(4)$ are depicted in Figure 9.

If this interpretation is correct-further studies are needed to answer this questionthen one could construct a continuum limit at a transition without order parameter. Since there is no transition with order parameter for all reducible lattice Thirring models with $N_{\mathrm{r}} \geq 1$, such an unusual transition is needed to relate the lattice models to continuum physics. Transitions without local order parameter have been reported previously in strongly coupled Fermi systems [39]. For unrealistically small $N_{\mathrm{r}} \lesssim 0.76$, there is one phase transition with order parameter. Actually, in [24] the maximum of the parity-invariant condensate has been measured and the results show clearly that a condensate forms at these small $N_{\mathrm{r}}$. 


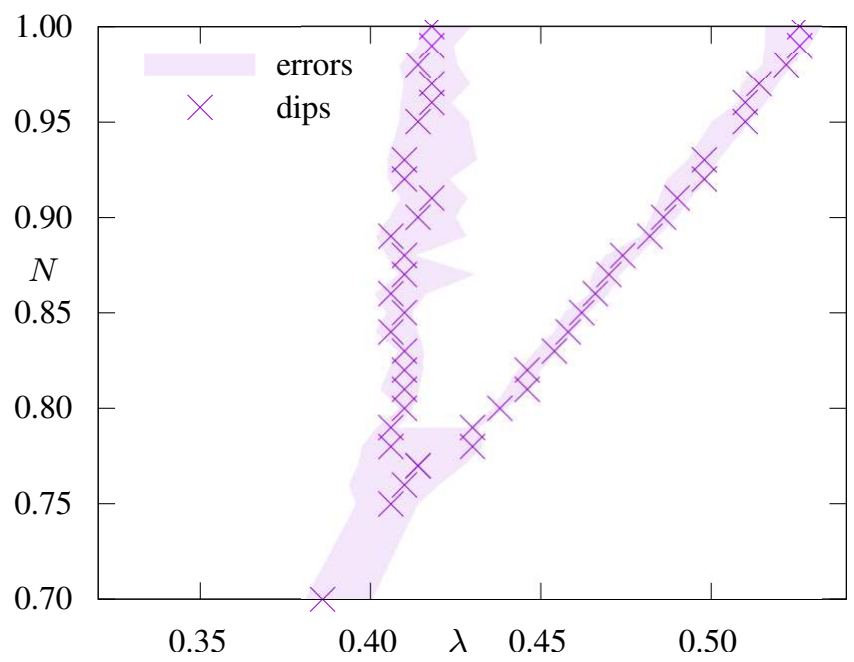

Figure 9. The dip positions of the susceptibility $\partial_{\lambda} k$.

\section{Summary}

In this paper, we reviewed our current knowledge about spontaneous symmetry breaking in 1+2D Thirring models. For odd irreducible flavor numbers, these models spontaneously break parity symmetry below:

$$
N_{\mathrm{f}}^{\text {crit }} \approx 9 \text {. }
$$

For $N_{\mathrm{f}}=1$, previously unpublished values from [36] for the critical couplings and critical exponents where given in (56) and (57).

We have collected strong evidence that the critical flavor number of the reducible Thirring models is below $N_{\mathrm{r}}=1$. We calculated the spectral density, the spectrum of scalar and pseudo-scalar mesons as function of the flavor number $N_{\mathrm{r}}$ between 0.5 and 1.0 and the maximum of the chiral condensate $[23,24]$. As a result, we find a critical flavor number,

$$
N_{\mathrm{r}}^{\text {crit }}=0.80(4) \text {. }
$$

In particular, we spotted two Goldstone bosons only for $N_{\mathrm{r}} \leq N_{\mathrm{r}}^{\text {crit }}$. Since a noninteger value of $N_{\mathrm{r}}$ probably does not describe a local quantum field theory, we conclude that there is no SSB in all reducible Thirring models. The critical value extracted from a combined analysis of all available data is a bit higher than the value 0.78 extracted from the susceptibility alone, but the two values 0.80 and 0.78 are compatible within the quoted statistical errors.

Simulations based on DWF with a large extra dimension spot a second order phase transition for one reducible flavor. From a fit to the equation of state the critical exponents $\delta=4.17(5)$ and $\eta=0.320(5)$ have been estimated [40]. Similar values have been extracted by the same authors in a previous study [26]. With DWF, a bilinear condensate forms at the transition, in contrast to the results obtained with chiral SLAC fermions. On the other hand, in simulations with DWF no transition without order parameter, as monitored by the fermionic 8-point function in Figure 8, is reported. With ongoing simulations this issue will hopefully be settled in the near future.

To correctly interpret the lattice results, one must stay away from the lattice-artifact phase, which in the dual formulation is well understood; as for gauge theories at large chemical potential there is Pauli-blocking on the lattice sites if the Thirring coupling exceeds a critical value. Our results are based on dedicated simulations with chiral SLAC fermions which respect all global inner symmetries and discrete space-time symmetries, such that there is no doubt that the lattice models represent the Thirring models in the continuum. One of the most pressing problem is the nature of the newly found (probably second order) phase transition without order parameter. We hope to report on this issue in a future work. 


\begin{abstract}
Author Contributions: Conceptualization, A.W.W. and J.J.L.; formal analysis, A.W.W. and J.J.L.; funding acquisition, A.W.W. and J.J.L.; methodology, A.W.W. and J.J.L.; project administration, A.W.W. and J.J.L.; supervision, A.W.W. and J.J.L.; writing-original draft preparation, A.W.W. and J.J.L.; writing-review and editing, A.W.W. and J.J.L. All authors have read and agreed to the published version of the manuscript.
\end{abstract}

Funding: This research was funded by the DFG graduate school GRK 1523/2.

Acknowledgments: We would like to thank the organizers of the workshop "Relativistic Fermions in Flatland: theory and application" "Quantum Theory and Symmetries", Simon Hands, Holger Gies, John Gracey and Igor Herbut for organizing an inspiring online meeting at ETC*-Trento. We thank our former co-workers Daniel Schmidt and Bjoern Wellegehausen who contributed considerably to the results presented in this publication.

Conflicts of Interest: The authors declare no conflict of interest.

\title{
References
}

1. Thirring, W. A soluble relativistic field theory. Ann. Phys. 1958, 3, 91-112. [CrossRef]

2. Klaiber, B. The Thirring model. In Boulder 1967; Lecture notes in Physics XA; Gordon and Breach: New York, NY, USA, 1968.

3. Sachs, I.; Wipf, A. Generalized Thirring models. Ann. Phys. 1996, 249, 380-429. [CrossRef]

4. Gies, H.; Janssen, L. UV fixed-point structure of the three-dimensional Thirring model. Phys. Rev. D 2010, 82, 085018. [CrossRef]

5. Wipf, A. Statistical Approach to Quantum Field Theory, 2nd ed.; Lecture Notes in Physics; Springer: Berlin/Heidelberg, Germany, 2021.

6. Gehring, F.; Gies, H.; Janssen, L. Fixed-point structure of low-dimensional relativistic fermion field theories: Universality classes and emergent symmetry. Phys. Rev. D 2015, 92, 085046. [CrossRef]

7. Senechal, D.; Tremblay, A.M.; Bourbonnais, C. (Eds.) Theoretical Methods for Strongly Correlated Electrons; CMR Series in Mathematical Physics; Springer: New York, NY, USA, 2013.

8. Troyer, M.; Wiese, U.J. Computational Complexity and Fundamental Limitations to Fermionic Quantum Monte Carlo Simulations. Phys. Rev. Lett. 2005, 94, 170201. [CrossRef]

9. Hoefling, F.; Nowak, C.; Wetterich, C. Phase transition and critical behaviour of the $\mathrm{d}=3$ Gross-Neveu model. Phys. Rev. B 2002, 66, 205111. [CrossRef]

10. Gomes, M.; Mendes, R.S.; Ribeiro, R.F.; da Silva, A.J. Gauge structure, anomalies, and mass generation in a three-dimensional Thirring model. Phys. Rev. D 1991, 43, 3516. [CrossRef]

11. Hong, D.K.; Park, S.H. Large-N analysis of (2+1)-dimensional Thirring model. Phys. Rev. D 1994, 49, 5507. [CrossRef]

12. Itoh, T.; Kim, Y.; Sugiura, M.; Yamawaki, K. Thirring model as a gauge theory. Progr. Theor. Phys. 1995, 93, 417-439. [CrossRef]

13. Sugiura, M. Fermion mass generation in the d-dimensional Thirring models as a gauge theory. Progr. Theor. Phys. 1997, 97, 311-326. [CrossRef]

14. Kondo, K.-I. Thirring model as a gauge theory. Nucl. Phys. B 1995, 450, 251-266. [CrossRef]

15. Ahn, Y.M.; Park, Q.H. Spontaneous breaking of generalized parity in 2+1-dimensional four-Fermi interactions. J. Korean Phys. Soc. 1998, 33, 655-658.

16. Hyun, S.J.; Lee, G.H.; Yee, J.H. Gaussian approximation of the $(2+1)$ dimensional Thirring model in the functional Schrödinger picture. Phys. Rev. D 1994, 50, 6542. [CrossRef] [PubMed]

17. Janssen, L.; Gies, H. Critical behavior of the (2+1)-dimensional Thirring model. Phys. Rev. D 2012, 86, 105007. [CrossRef]

18. Dabelow, L.; Gies, H.; Knorr, B. Momentum dependence of quantum critical Dirac systems. Phys. Rev. D 2019, 99, 125019. [CrossRef]

19. del Debbio, L.; Hands, S.J.; Mehegan, J.C. The three dimensional Thirring model for small Nf. Nucl. Phys. B 1997, 502, 269-308. [CrossRef]

20. del Debbio, L.; Hands, S.J. The three-dimensional Thirring model for Nf $=4$ and Nf $=6$. Nucl. Phys. B 1999, 552, 339-362. [CrossRef]

21. Hands, S.; Lucini, B. The phase diagram of the three dimensional Thirring model. Phys. Lett. B 1999, 461, 263-269. [CrossRef]

22. Christofi, S.; Hands, S.; Strouthos, C. Critical flavor number in the three dimensional Thirring model. Phys. Rev. D 2007, 75, 101701. [CrossRef]

23. Wellegehausen, B.; Schmidt, D.; Wipf, A. Critical flavour number of the Thirring model in three dimensions. Phys. Rev. D 2017, 96, 094504. [CrossRef]

24. Lenz, J.J.; Wipf, A.; Wellegehausen, B. Absence of chiral symmetry breaking in Thirring models in $1+2$ dimensions. Phys. Rev. D 2019, 100, 054501. [CrossRef]

25. Hands, S. Critical flavor number in the $2+1$ D Thirring model. Phys. Rev. D 2019, 99, 034504. [CrossRef]

26. Hands, S.; Mesiti, M.; Worthy, J. Critical behavior in the single flavor Thirring model in 2 + 1D. Phys. Rev. D 2020, 102, 094502 . [CrossRef]

27. Wozar, C.; Wipf, A. Supersymmetry breaking in low dimensional models. Ann. Phys. 2012, 327, 774-807. [CrossRef] 
28. Bergner, G.; Kaestner, T.; Uhlmann, S.; Wipf, A. Low-dimensional supersymmetric lattice models. Ann. Phys. 2008, 323, 946-988. [CrossRef]

29. Schmidt, D. Three-Dimensional Four-Fermion Theories with Exact Chiral Symmetry on the Lattice. Ph.D. Thesis, FriedrichSchiller-University Jena, Jena, Germany, 2018; doi:10.22032/dbt.34148. [CrossRef]

30. Lang, T.C.; Läuchli, A.M. Quantum Monte Carlo Simulation of the Chiral Heisenberg Gross-Neveu-Yukawa Phase Transition with a Single Dirac Cone. Phys. Rev. Lett. 2019, 123, 137602. [CrossRef]

31. Lenz, J.; Pannullo, L.; Wagner, M.; Wellegehausen, B.; Wipf, A. Inhomogeneous phases in the Gross-Neveu model in $1+1$ dimensions. Phys. Rev. D 2020, 101, 094512. [CrossRef]

32. Lenz, J.; Mandl, M.; Wipf, A. Inhomogeneities in the 2-Flavor Chiral Gross-Neveu Model. arXiv 2021, arXiv:2109.05525.

33. Karsten, L.H.; Smit, J. The vacuum polarization with SLAC lattice fermions. Phys. Lett. B 1979, 85, 100-102. [CrossRef]

34. Hanada, M.; Nishimura, J.; Takeuchi, S. Non-lattice simulation for supersymmetric gauge theories in one dimension. Phys. Rev. Lett. 2007, 99, 161602. [CrossRef]

35. Chandrasekharan, S. Fermion bag approach to lattice field theories. Phys. Rev. D 2010, 82, 025007. [CrossRef]

36. Lenz, J.J. Spontaneous Symmetry Breaking in Four Fermion Theories. Master's Thesis, Friedrich-Schiller-University Jena, Jena, Germany, 2018. Available online: http:/ / www.tpi.uni-jena.de/qfphysics/homepage/wipf/theses.html (accessed on 22 December 2021).

37. Haldane, F.D.M. Model for a Quantum Hall Effect without Landau Levels: Condensed-Matter Realization of the "Parity Anomaly". Phys. Rev. Lett. 1988, 61, 2015. [CrossRef] [PubMed]

38. Verbaarschot, J.J.M.; Wettig, T. Random Matrix Theory and Chiral Symmetry in QCD. Ann. Rev. Nucl. Part. Sci. 2000, 50, 343-410. [CrossRef]

39. Catterall, S.; Schaich, D. Novel phases in strongly coupled four-fermion theories. Phys. Rev. D 2017, 96, 034506. [CrossRef]

40. Hands, S.; Mesiti, M.; Worthy, J. Critical Behaviour in the Single Flavor Planar Thirring Model. In Proceedings of the 38th International Symposium on Lattice Field Theory, Cambridge, MA, USA, 26-30 July 2021. 